\title{
Molecular pathways underlying inhibitory effect of antimicrobial peptide Nal-P-113 on bacteria biofilms formation of Porphyromonas gingivalis W83 by DNA microarray
}

\author{
Hong-yan Wang', Li Lin', Li-si Tan', Hui-Yuan Yu², Jya-Wei Cheng ${ }^{2}$ and Ya-ping Pan ${ }^{\text {** }}$
}

\begin{abstract}
Background: Wound-related infection remains a major challenge for health professionals. One disadvantage in conventional antibiotics is their inability to penetrate biofilms, the main protective strategy for bacteria to evade irradiation. Previously, we have shown that synthetic antimicrobial peptides could inhibit bacterial biofilms formation.

Results: In this study, we first delineated how Nal-P-113, a novel antimicrobial peptide, exerted its inhibitory effects on Porphyromonas gingivalis W83 biofilms formation at a low concentration. Secondly, we performed gene expression profiling and validated that Nal-P-113 at a low dose significantly down-regulated genes related to mobile and extrachromosomal element functions, transport and binding proteins in Porphyromonas gingivalis W83.

Conclusions: These findings suggest that Nal-P-113 at low dose is sufficient to inhibit the formation of biofilms although Porphyromonas gingivalis W83 may maintain its survival in the oral cavity. The newly discovered molecular pathways may add the knowledge of developing a new strategy to target bacterial infections in combination with current first-line treatment in periodontitis.
\end{abstract}

Keywords: Porphyromonas gingivalis W83, Nal-P-113, Microarray, Biofilms

\section{Backgrounds}

Periodontitis is one of the worldwide infectious diseases in humans, with approximately half of the adults experiencing some degrees of chronic periodontitis in developing countries, while $15 \%$ of United Kingdom population have developed severe periodontitis [1]. With an increase in aging population, the problem becomes more critical because elderly patients have compromised immune systems which predispose them to a higher risk of contracting bacterial infections. Reduced ability in tissue repairing further substantiates the problem. Antibiotics have been proven to work effectively against bacterial

\footnotetext{
* Correspondence: yppan@cmu.edu.cn

${ }^{1}$ Department of Periodontics, School of Stomatology, China Medical

University, Shenyang 110002, China

Full list of author information is available at the end of the article
}

infections. However, the overuse of drugs clearly drives the evolution of bacteria resistance, endangering the efficacy of antibiotics. Therefore there is an emergent need to identify novel compounds to counteract bacterial infections. In general, conventional antibiotics are unable to penetrate biofilms. The formation of biofilms allows the bacteria to anchor and propagate in the tissue. Therefore, targeting the formation of biofilms may be a new therapeutic option for periodontitis.

Previous studies have shown that synthetic antimicrobial peptides inhibit bacterial biofilms formation. Numerous studies have confirmed that the major antimicrobial peptides mediated bactericidal mechanism is via rapid perforation of the cell membrane as well as activation of the apoptotic program by interrupting the normal physiological metabolism [2-4]. It has been demonstrated that 
antimicrobial peptide LL-37-treated Pseudomonas aeruginosin showed enormous changes in its gene transcription, with many de-regulated genes involved in the function of flagellar [5]. Similarly, antimicrobial peptide 1037 treatment for $24 \mathrm{~h}$ significantly changed the gene expression profiles knowing to be regulated by LL-37 treatment [6]. Nal-P-113, a modified version of antimicrobial peptide P-113, its amino sequence is AKR-Nal-NalGYKRKF-Nal-NH2. Antimicrobial peptide P-113 showed promising antimicrobial effects against a variety of pathogens [7-11]. Compared to P-113, Nal-P-113 maintained its effects when exposed to a high salt concentration and therefore it was an ideal candidate for application in complicated matrices including oral cavity, serum and plasma [12].

We have previously shown that Nal-P-113 exerts its anti-bactericidal effects in a rat periodontitis model with a significant reduction in tissue inflammation. Furthermore, we have found that Nal-P-113 inhibits Porphyromonas gingivalis ( $P$. gingivalis) W83 biofilms formation. In this study, we aimed to determine the effects of different concentrations of Nal-P-113 in biofilms formation. Besides, we performed gene expression profiles in Nal-P113-treated $P$. gingivalis W83 to delineate the underlying molecular mechanism of Nal-P-113-inhibited biofilms formation.

\section{Methods}

\section{Bacteria strain}

P. gingivalis W83 was a gift from Professor RJ Lamont (now in Department of oral Immunology and Infectious Disease, School of Dentistry, University of Louisville) from College of Dentist, University of Florida. Freshly prepared brain heart infusion (BHI, Difco Laboratories, MI, USA) agar medium supplemented with $5 \%$ sterile defibrinated sheep's blood, $1 \%$ hemin, and $0.1 \%$ menadione, was used to grow $P$. gingivalis $\mathrm{W} 83$ at $37^{\circ} \mathrm{C}$ under anaerobic conditions $\left(80 \% \mathrm{~N}_{2}, 10 \% \mathrm{H}_{2}\right.$ and $\left.10 \% \mathrm{CO}_{2}\right)$ for 5 to 7 days.

\section{Reagents}

Antimicrobial peptide Nal-P-113, Ac-AKR-Nal-NalGYKRKF-Nal- $\mathrm{NH}_{2}$, was provided by Prof. Jiawei Cheng in National Tsing Hua University [13]. $\mathrm{H}_{2} \mathrm{O}_{2}$ was purchased from Sigma Aldrich (CA).

\section{Bactericidal assay}

P. gingivalis W83 was diluted to $5 \times 10^{5} \mathrm{CFU} / \mathrm{mL}$ (CFU, colony forming units). The bacteria were treated with Nal-P-113 in $100 \mu \mathrm{L}$ culture medium for $24 \mathrm{~h}$. Then an aliquot $(50 \mu \mathrm{L})$ of the resulting bacterial cell suspension was cultivated on a brain heart infusion agar plate. The bacterial cells were enumerated after incubation at $37{ }^{\circ} \mathrm{C}$ for 7 days. All experiments were repeated three times.

\section{Growth inhibition assay}

P. gingivalis W83 culture was diluted to $5 \times 10^{5} \mathrm{CFU} /$ $\mathrm{mL}$. The bacteria were treated with Nal-P-113 at different concentrations $(0,5,10,20,40,80,160$ and $320 \mu \mathrm{g} /$ $\mathrm{mL}$ respectively) in $100 \mu \mathrm{L}$ culture medium for $48 \mathrm{~h}$. The cell growth was measured by the absorbance at $600 \mathrm{~nm}$ in a microplate reader (Tecan Infini M200, Switzerland). All experiments were repeated three times.

\section{Scanning electron microscopy (SEM) analysis on Biofilms} Biofilms formation was quantified on 6-well plates (Corning, Netherlands) which were coated with artificial saliva (Guangzhou Kodak Adhesives Co. Ltd., China). Five hundred microliter of $P$. gingivalis W83 $\left(5 \times 10^{6} \mathrm{CFU} / \mathrm{mL}\right)$ with or without $6.25 \mu \mathrm{g} / \mathrm{mL}$ Nal-P-113 treatment was dropped on 6-well plates and cultured for $48 \mathrm{~h}$ to establish biofilms. Then, the samples were fixed with $2.5 \%$ glutaraldehyde (BioChemika, Fluka, USA), washed with PBS and gradually dehydrated with ethanol. The processed samples were smeared onto copper plates followed by gold sputtering, and images were acquired using scanning electron microscopy (Inspect F50, FEI Company, USA) at 20,000 × magnification.

\section{Microarray hybridization}

The total RNA of the bacteria treated with or without $6.25 \mu \mathrm{g} / \mathrm{mL}$ Nal-P-113 was extracted, converted to cDNA, labeled with Cy3-dCTP (GE, Healthcare, CA) and followed by hybridization with $P$. gingivalis W83 chip (Agilent, CA). Array hybridization, washing, scanning and data analysis were performed at the CapitalBio Corporation (Beijing, China).

\section{Data analysis}

The array data were analyzed with the GeneSpring software V12 (Agilent). Significant genes were defined by following criterions: $\mathrm{FDR}<5 \%$ and fold change $>2[14,15]$.

\section{Quantitative PCR (qPCR)}

The total RNA of $P$. gingivalis W83 treated with or without $6.25 \mu \mathrm{g} / \mathrm{mL}$ Nal-P-113 was reversed transcribed into cDNA with the M-MLV RTase cDNA Synthesis Kit (Takara, China). Real-time quantitative PCR reaction was performed using SYBR Premix Ex TaqTM II PCR Master Mix Reagents Kit (Takara). The primers for the RT-qPCR were listed in Additional file 1: Table S1. qPCR was performed three times for each sample. Relative quantification of the mRNA levels was performed using the comparative $\mathrm{Ct}$ method with the formula $2^{-\Delta \Delta \mathrm{Ct}}$.

\section{Hydrogen peroxide pre-treatment in $P$. gingivalis}

$P$. gingivalis W83 was treated with $\mathrm{H}_{2} \mathrm{O}_{2}$ at different concentrations $(0,0.5,1$, and $3 \mathrm{mM}$, respectively) for $1 \mathrm{~h}$. The $\mathrm{H}_{2} \mathrm{O}_{2}$ was washed away with phosphate buffer 
saline before subsequent experiments. Total RNA of the respective $\mathrm{H}_{2} \mathrm{O}_{2}$-treated $P$. gingivalis was extracted and followed by RT-qPCR to determine the mRNA expression of PG0841, PG0842, PG0872, PG0874, PG0875, PG1473, PG1474, PG1475, PG1478, PG1479, PG1482 and PG1485.

\section{Biofilms susceptibility assay}

$3 \mathrm{mM} \mathrm{H}_{2} \mathrm{O}_{2}$ was used to change transposase genes expression of $P$. gingivalis W83 for $1 \mathrm{~h}$. The effect of Nal-P-113 on P. gingivalis W83 biofilms formation was examined using the microdilution method [12]. The resulting biofilms were fixed with $95 \%$ methanol and stained with $0.5 \%(\mathrm{w} / \mathrm{v})$ crystal violet prior dissolving with $95 \%$ ethanol and subjected to microplate reader at absorption $590 \mathrm{~nm}$. Percentage of inhibition was calculated using the equation [1-(A590 of the test/A590 of non-treated control) $] \times 100$. All experiments were repeated three times.

\section{Enzyme linked immunosorbent assay (ELISA)}

Lipopolysaccharide, free hemin and hemoglobin were measured by commercial ELISA kit according to the respective manual. Free hemin ELISA kit was purchase from Abnova, Taiwan, and the hemoglobin ELISA kit was purchased from Leagene, China. The measurements were analysis by Curve Expert $1.3(\mathrm{AL})$.

\section{Statistical analysis}

All experiments were performed in triplicate and repeated at least three times. Data were expressed as means \pm standard deviations (SD). ANOVA and independent samples $t$-test were used to calculate the significance among the groups (SPSS Inc., IL, USA). $P$-value $<0.05$ was considered statistically significant.

\section{Results}

The antibacterial activity of Nal-P-113 against $P$. gingivalis W83

$P$. gingivalis W83 is one of the major pathogenic species in dental plaque biofilms. Firstly, we examined the inhibitory effects of Nal-P-113 on P. gingivalis W83 biofilms formation. Consistent with our previous study, $6.25 \mu \mathrm{g} / \mathrm{mL}$ Nal-P-113 significantly inhibited $P$. gingivalis W83 biofilms formation (Fig. 1a). The biofilms inhibitory effect of Nal-P-113 was further validated with scanning electron microscopy (SEM) (Fig. 1b). Before treated with Nal-P-113, the bacteria engaged with each other and resulted in the formation of biofilms. Upon $6.25 \mu \mathrm{g} / \mathrm{mL}$ Nal-P-113 treatment, the bacteria continued to divide and were unable to fuse together, therefore inhibited the formation of biofilms. Conversely, treatment with $320 \mu \mathrm{g} / \mathrm{mL}$ Nal-P-113 resulted in a complete breakdown of the bacteria structure. Secondly, we evaluated the antibacterial activity of Nal-P-113 against planktonic $P$. gingivalis W83. Results showed that Nal-P113 at a low concentration exhibited neither bactericidal activity nor growth inhibitory effect against $P$. gingivalis W83 (Fig. 1c, d). These results suggested that Nal-P-113 at high concentrations inhibited biofilms formation by direct killing of the bacteria, whereas Nal-P-113 at a low concentration adopted an alternative mechanism to inhibit the formation of biofilms in P. gingivalis W83.

\section{Nal-P-113 induced differential gene expression and disrupted the signaling pathways in $P$. gingivalis W83}

To further understand the underlying mechanism of Nal-P-113-inhibited biofilms formation in $P$. gingivalis W83, we exposed the $P$. gingivalis W83 with $6.25 \mu \mathrm{g} / \mathrm{mL}$ Nal-P-113 for $0.5 \mathrm{~h}$ prior subjected to microarray gene analysis. Genes with expression fold change greater than 2 and FDR $<5 \%$ were identified as dysregulated genes. Supervised clustering analysis showed that Nal-P-113 treatment induced a significant alternation of gene expression in $P$. gingivalis W83 compared to untreated control (Fig. 2a). In total, Nal-P-113 treatment resulted in an increase in 204 transcripts and a decrease in 220 transcripts in P. gingivalis W83 (Additional file 1: Table S2). The complete list of gene expression values has been deposited in NCBI's Gene Expression Omnibus (http://www.ncbi.nlm.nih.gov/geo/query/ acc. . gi? acc $=$ GSE 93873).

Although the genome of $P$. gingivalis W83 was published more than a decade ago, little is known about the interconnections among signaling pathways and how the interplay may impact the therapeutic interventions. We input the genes that were differentially expressed after Nal-P-113 treatment to STRING database for KEGG pathway analysis. Differentially regulated genes can be categorized into six groups according to the TIGR genome database. Majority of the dysregulated genes were involved in one of the following cellular functions/pathways: mobility and extrachromosomal element functions, energy metabolism, cell envelope, transport and binding proteins, protein synthesis and protein fate. Interestingly, dysregulated genes that were found to be involved in the down-regulated pathways were mainly related to mobility, extrachromosomal element functions as well as transport and binding protein. Meanwhile, differentially expressed genes involved in the up-regulated pathways were associated to cell envelope, energy metabolism, protein fate and synthesis (Fig. $2 \mathrm{~b}$ and c).

Following, we selected the top 12 up-regulated and down-regulated genes (Additional file 1: Table S3) from the microarray analysis for validation. Apart from the dysregulated genes, we also included four additional genes whose expressions were not altered by Nal-P-113 as negative control. All top 6 up-regulated and down- 

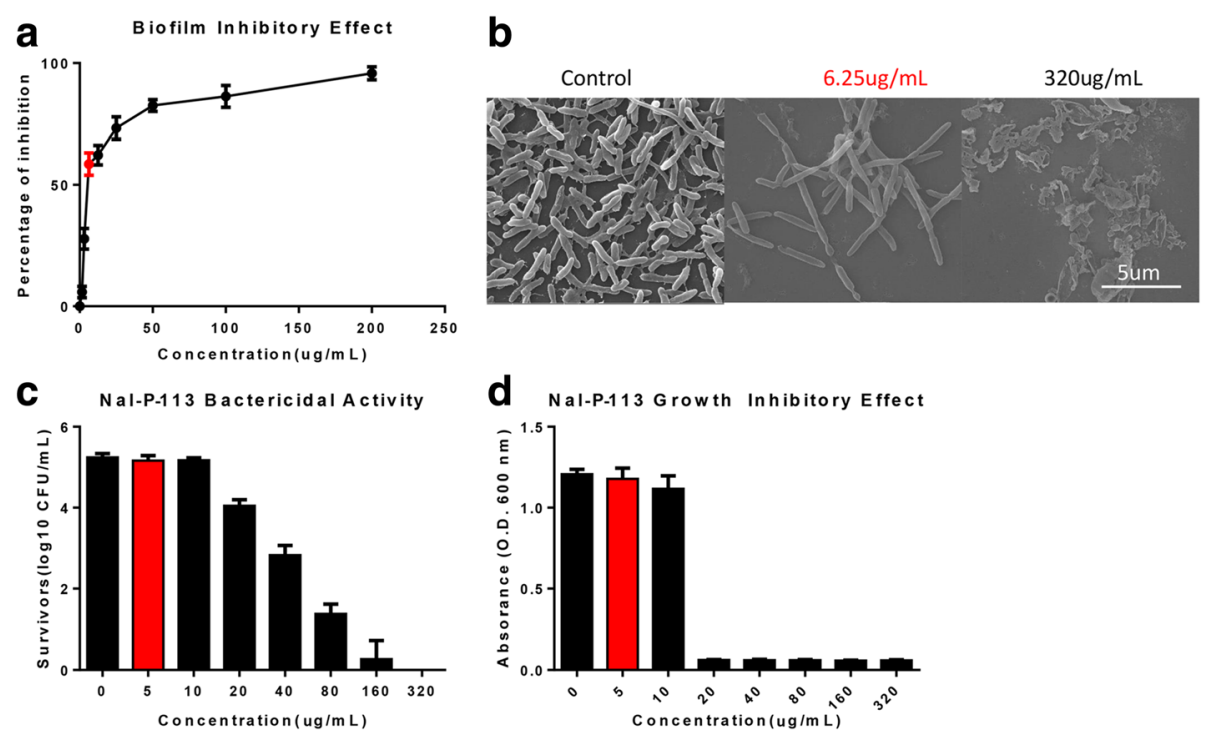

Fig. 1 The MIC of Nal-P-113 to P. gingivalis W83 was $160 \mu \mathrm{g} / \mathrm{mL}$, the MBC was $20 \mu \mathrm{g} / \mathrm{mL}$, the MBIC was $6.25 \mu \mathrm{g} / \mathrm{mL}$. The above results showed that Nal-P-113 inhibited P. gingivalis biofilms at a concentration that did not inhibit $P$. gingivalis growth. a Biofilms susceptibility assay of Nal-P-113 against $P$. gingivalis W83. $\mathbf{b}$ SEM image of $P$. gingivalis W83 in biofilm formation. $\mathbf{c}$ Bacterial growth assay of Nal-P-113-treated $P$. gingivalis W83. $\mathbf{d}$ Bactericidal assay of Nal-P-113-treated $P$. gingivalis W83. Data shown here are the mean \pm S.D. from three independent cultures

regulated genes showed a similar trend and fold change in expression levels between microarray analysis and quantitative PCR, with a correlation value (R) of 0.964 (Fig. 2d and e).

\section{The biological effect of dysregulated genes and pathways after Nal-P-113 treatment}

To further verify the underlying mechanism in Nal-P113-treated $P$. gingivalis W83, we first used ELISA to detect free hemin and hemoglobin in liquid BHI. In accordance with the down-regulation of hmuY (PG1551) and HmuR (PG1552), known as proteins involved in the uptake of nutrients in a bacterial system (Fig. 3a), we observed a significant decrease in heme but not hemoglobin in the Nal-P-113-treated $P$. gingivalis W83 (Fig. $3 \mathrm{~b}$ and c). Besides, when $3 \mathrm{mM} \mathrm{H} \mathrm{H}_{2} \mathrm{O}_{2}$ induced the expression of the transpoases genes that were found to be down-regulated by Nal-P-113 (Fig. 3d), $6.25 \mu \mathrm{g} / \mathrm{mL}$ Nal-P-113 did not inhibit P. gingivalis W83 biofilms formation (Fig. 3e). The result suggested that the transpoase pathways played a role in the Nal-P-113-regulated biofilms formation in P. gingivalis W83.

\section{Discussion}

P. gingivalis is a Gram-negative oral anaerobe that has been known to play a major role in the pathogenesis of periodontitis. $P$. gingivalis, presents within the forming biofilms, orchestrates the virulence of the biofilms and consequent tissue inflammatory response to destroy the gingival tissues and ultimately resulting in tooth loss. Previously, we and others have shown that synthetic antimicrobial peptides can inhibit bacterial biofilms formation [12]. Multiple lines of evidence suggested that antimicrobial peptides acted via rapid perforation of the cell membrane [2-4]. Nevertheless, our SEM image showed that the cell membrane structure remained intact after treatment of $6.25 \mu \mathrm{g} / \mathrm{mL}$ Nal-P-113, indicating that a low concentration of Nal-P-113 did not disrupt the bacteria membrane. Nevertheless, Nal-P-113 at a low dose may still have indirect effects on bacterial membrane. For example, PG0192 and PG0193, encoding the cationic outer membrane proteins that involve in the colonization and biofilms formation, were both downregulated by 2.15 fold and 2.07 fold, respectively, upon Nal-P-113 treatment. In parallel, PG2133 encoding lipoprotein was down-regulated by 2.50 fold. The Gramnegative bacterial lipoprotein is an essential component of the outer membrane, which plays a key role in bacterial infection, survival, as well as invading and damaging the host's immune defense. The down-regulation of PG0192, PG0193 and PG2133 may be correlated with the effects of antimicrobial peptides in the bacterial membranes that reduce their ability to maintain cellular structure, to form biofilms or to survive.

Studies have reported the inhibiting effects of antimicrobial peptides in biofilms formation, which involved the disruption of the bacteria's physiological metabolism [2-4]. However, our gene expression profiling demonstrated that the presence of Nal-P-113 enhanced $P$. gingivalis W83 aerobic respiration to survive. There were 23 differentially expressed genes function in energy metabolism, five genes were down-regulated, and 18 


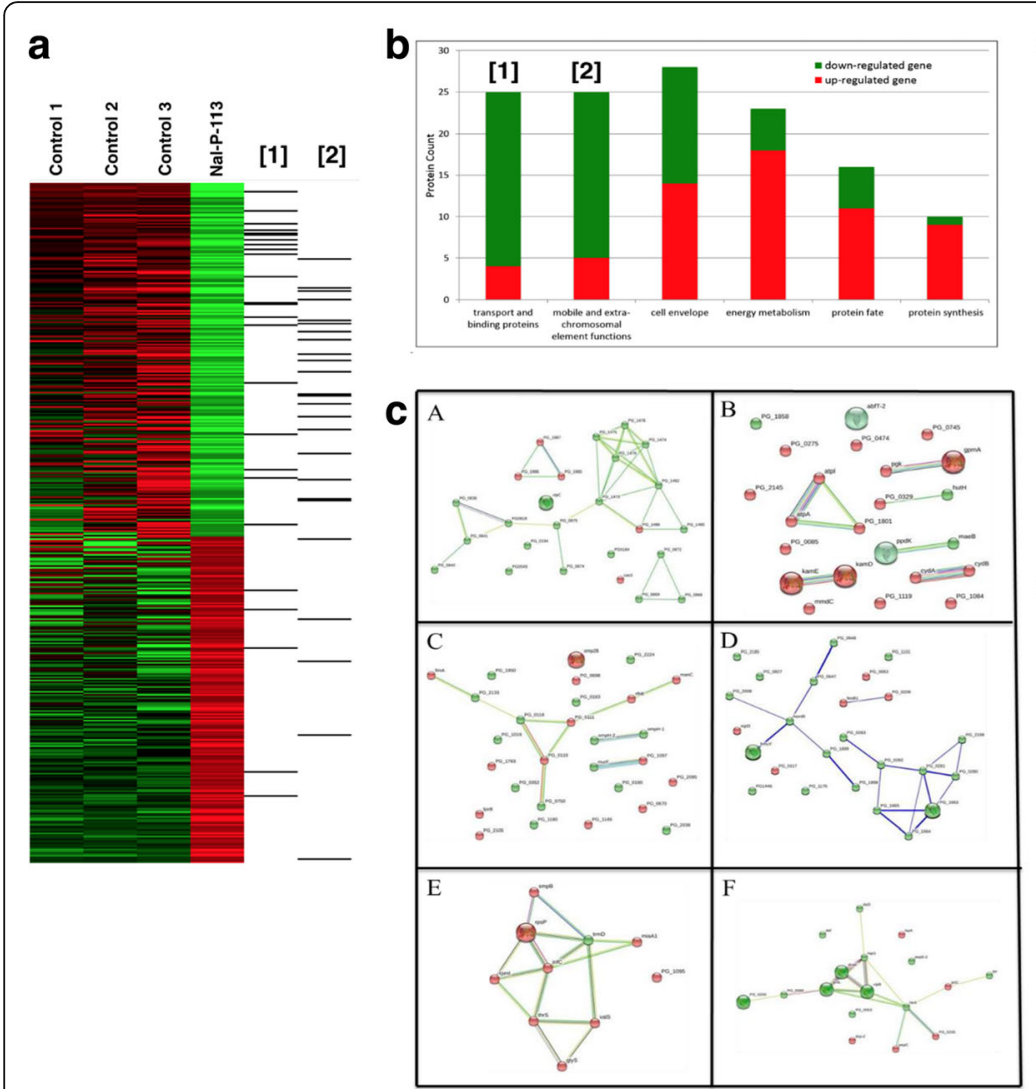

d P.gingivalis W 83
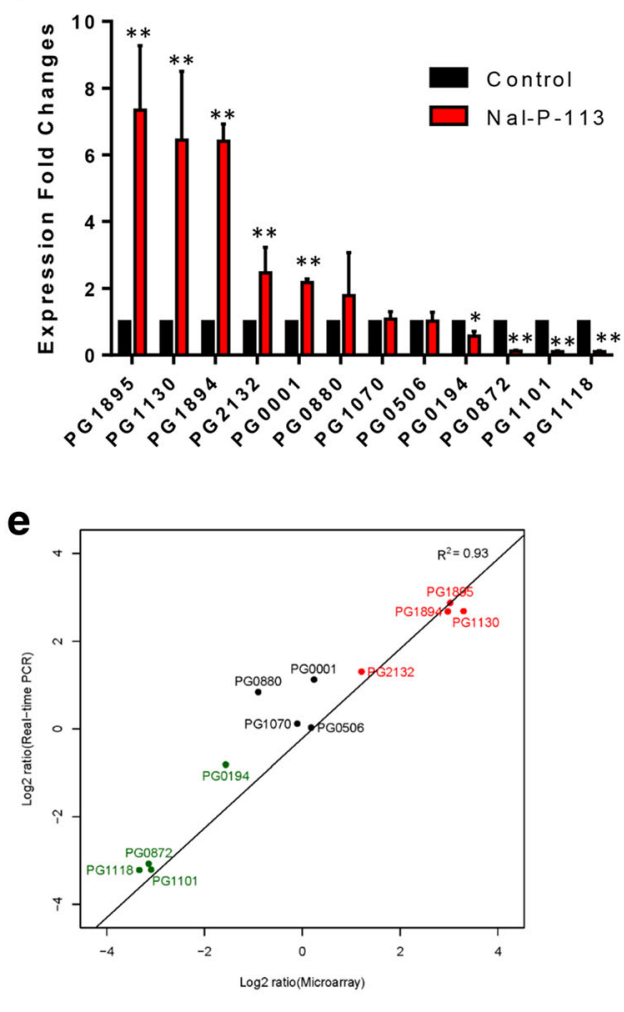

Fig. 2 Differentially gene expression was exhibited in Nal-P-113-treated Porphyromonas gingivalis W83 as shown in KEGG pathway analysis. Differentially regulated genes can be categorized into six groups according to the TIGR genome database, including mobility and extrachromosomal element functions, energy metabolism, cell envelope, transport and binding proteins, protein synthesis and protein fate. 12 genes were selected to verify the consistency of quantitative PCR and Microarray analysis. a Supervised clustering of Nal-P-113-treated and non-treated Porphyromonas gingivalis $(n=3) ;(\mathbf{b})$ KEGG pathway analysis on the deferentially expressed genes in Nal-P-113-treated Porphyromonas gingivalis. c The network was constructed in the six major dys-regulated pathways. $a$ : Differentially expressed genes related to mobile and extrachromosomal element functions. $b$ : Differentially expressed genes related to energy metabolism. c: Differentially expressed genes related to cell envelope. $d$ : Differentially expressed genes related to transport and binding protein. e: Differentially expressed genes related to protein synthesis. f. Differentially expressed genes related to protein fate. Nodes and edges represent differentially expressed genes and interactions among them. Up-regulated genes were represented as pink nodes, down-regulated genes were represented as green nodes. $\mathbf{d}$ The fold changes of gene expression levels in Porphyromonas gingivalis after Nal-P-113 treatment. Data shown here are the mean \pm S.D. from three independent cultures. ${ }^{*}, P<0.05$; ${ }^{* *}, P<0.01$. e Correlation of the expression fold change between quantitative $P C R$ and microarray. Green, down-regulated genes, Black, unaffected genes, Red, up-regulated genes

genes were up-regulated. Cytochrome dubiquinol oxidaseencoding genes, cydA (PG0900) and cydB (PG0899), which are related to aerobic respiration in $P$. gingivalis [16], were up-regulated by 3.64-fold and 2.06-fold, respectively. Within the locus encoding cydA and cydB, there is an open reading frame encoding a protein of unknown function (PG0901), and was up-regulated in the presence of Nal-P-113, indicating that it may be a member of the same transcriptional unit as the cydAB operon. PG0130, encoding the phosphoglycerate mutase that participates in DNA and RNA synthesis, was up-regulated. Furthermore, genes related to protein synthesis were upregulated. PG0502, encoding SsrA-binding protein, was up-regulated by 2.61-fold. PG0992, encodes threonyltRNA synthetase, PG1132 encodes valyl-tRNA synthetase, and PG2165 encodes glycyl-tRNA synthetase, were found to be up-regulated. Ammonia acyl-tRNA synthetase is one of the key enzymes in protein synthesis [17]. PG2117 and PG0990 both encoding ribosomal proteins were shown to be up-regulated by 3.32-fold and 2.52-fold, respectively.

Moreover, transporting and binding protein pathway was also significantly inhibited by Nal-P-113 treatment. 4 out of the 25 transporting and binding protein-related genes were up-regulated, and the remaining 19 genes were down-regulated. PG0282 and PG1663 encode ABC transporter and ATP-binding protein, respectively. PG0281, PG0280, PG1664 and PG1665, encoding ABC transporter and permease proteins, were down-regulated. $\mathrm{ABC}$ transporters are a group of transmembrane proteins, known to be associated with a variety of physiological processes 


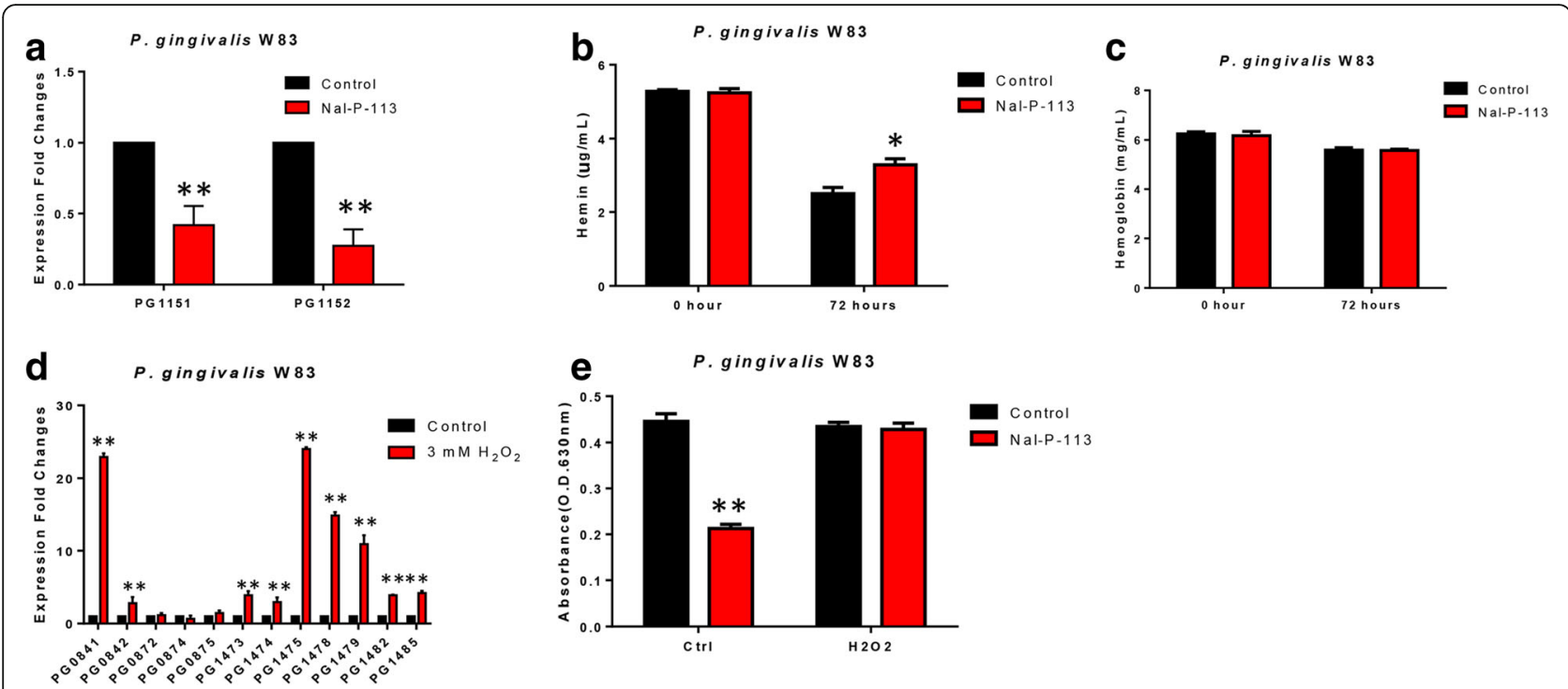

Fig. 3 Validation of biological effect according to gene expression profiling analysis. a The fold changes of PG1151 and PG1152 gene expression levels in Porphyromonas gingivalis after Nal-P-113 treatment. b Free hemin concentration in $P$. gingivalis W83 culture medium either with or without treatment of Nal-P-113. c Hemoglobin concentration in P. gingivalis W83 culture medium either with or without treatment of Nal-P-113. d The expression of transposases levels in Porphyromonas gingivalis after $3 \mathrm{mM} \mathrm{H}_{2} \mathrm{O}_{2}$ treatment for one hour. e Biofilm susceptibility assay of Nal-P-113-treated $P$. gingivalis W83 with or without $\mathrm{H}_{2} \mathrm{O}_{2}$ pretreatment. Data shown here are the mean \pm S.D. from three independent cultures, ${ }^{*}, P<0.05,{ }^{* *}, P<0.01$

including the uptake of nutrients, the non-classical secretion of signaling molecules and toxins, multidrug resistance and the development of human disease. $A B C$ transporters play a role in cell-to-surface and cell-to-cell interactions in biofilms development [18-20]. The observed differentially expressed genes in $\mathrm{ABC}$ transporter superfamily were in line with our SEM result, demonstrating the loss of cell contact in P. gingivalis W83 after treated with Nal-P-113 at a low concentration.

PG2016, encoding CRISPR-associated helicase, PG1985, PG1986 and PG1987 encoding other CRISPR-associated proteins, were shown to be up-regulated and may be related to the immune response due to foreign DNA. The down-regulation in integrase and the up-regulation of CRISPR-associated proteins indicated that the killing of $P$. gingivalis W83 by Nal-P-113 differed to that of antibiotics. Therefore, the acquisition of resistance to Nal-P-113 is less likely compared to antibiotic since different mechanisms are activated in the bacteria system.

Lastly, PG1551 encodes hmuY protein, while PG1552 encodes the TonB-dependent receptor HmuR. These two genes were down-regulated by 2.39 -fold and 3.67 fold, respectively. The bacterial uptake system for heme requires two kinds of proteins: HmuY, which scavenges heme from host hemoproteins, and HmuR, which transports nutrients across the bacterial cell membrane. The down-regulation of these two genes by Nal-P-113 suggested that Nal-P-113 treatment might weaken the ability of $P$. gingivalis W83 to utilize hemin. In fact, our ELISA assays verified that the concentration of hemin decreased significantly in the Nal-P-113-treated P. gingivalis W83 liquid BHI, indicating that Nal-P-113-treated bacteria could not utilize the hemin efficiently, therefore inhibited the formation of biofilms. Furthermore, PG0841, PG0842, PG0872, PG0874, PG0875, PG1473, PG1474, PG1475, PG1478, PG1479, PG1482, PG1485, which encode the transposase genes were all downregulated upon Nal-P-113 treatment. Previous studies have shown that $\mathrm{H}_{2} \mathrm{O}_{2}$ induces the expression of transposase genes in $P$. gingivalis [21,22]. Our results showed that $\mathrm{H}_{2} \mathrm{O}_{2}$ pre-treatment abolished $6.25 \mathrm{mg} / \mathrm{mL}$ Nal-P113 anti-biofilms formation in $P$. gingivalis W83, which indicated that transposase genes played an important role in Nal-P-113-mediated biofilms formation.

\section{Conclusions}

$P$. gingivalis is one of the major etiologic agents which contributes to chronic periodontitis. It attributes to the formation of subgingival biofilms and stimulates a cascade of inflammatory reaction. Our results showed that Nal-P113 exerted its anti-microbial and anti-biofilms effects in $P$. gingivalis W83 by mediating the energy metabolism, protein synthesis, mobile and extrachromosomal element functions, transport and binding proteins of the bacteria. In addition, we verified that Nal-P-113 acted through the down-regulation of transposase genes or reduction of hemin utilization in P. gingivalis W83 to inhibit the formation of biofilms. In summary, this study provides a molecular biological basis of Nal-P-113 in the prevention and treatment of periodontitis. 


\section{Additional file}

Additional file 1: Table S1. List of primers used for $\mathrm{QPCR}$ experiments. Table S2. Up-regulated gene list in Nal-P-113 treated P.gingivalis W83 (hypothetical protein excluded). Table S3. Down-regulated gene list in Nal-P-113 treated P.gingivalis W83 (hypothetical protein excluded). (DOCX 46 kb)

\section{Abbreviations}

BHI: Brain heart infusion; CFU: Colony forming units; CRISPR: Clustered regularly interspaced short palindromic repeat; ELISA: Enzyme linked immunosorbent assay; LPS: Lipopolysaccharide; qPCR: Quantitative PCR: SEM: Scanning electron microscopy

\section{Acknowledgements}

None.

\section{Funding}

This work was supported by the Natural Science Foundation of China (81470745 to YPP) and the Natural Science Foundation of Liaoning Province (2015020754 to HYW).

\section{Availability of data and materials}

All microarray data have been submitted to NCBI GEO (http://www.ncbi.nlm. nih.gov/geo/query/acc.cgi?acc=GSE 93873). The rest data and analyses are uploaded as an Additional file 1.

\section{Authors' contributions}

HYW carried out microarray analysis, analysed the data, and drafted the manuscript. LL performed $P$. gingivalis W83 culture and inoculation. LST performed real-time $P C R$ analysis and Elisa test. HYY was responsible for $P$. gingivalis growth inhibition assay and biofilm susceptibility assay. JWC synthesized Nal-P-113. YPP designed the study and drafted the manuscript. All authors read and approved the final manuscript.

\section{Competing interests}

The authors declare that they have no competing interests.

\section{Consent for publication}

Not applicable.

\section{Ethics approval and consent to participate}

Not applicable.

\section{Author details}

'Department of Periodontics, School of Stomatology, China Medical University, Shenyang 110002, China. ${ }^{2}$ Institute of Biotechnology and Department of Medical Science, National Tsing Hua University, Hsinchu 300, Taiwan.

\section{Received: 26 September 2016 Accepted: 7 February 2017}

\section{Published online: 17 February 2017}

\section{References}

1. Hussain M, Stover CM, Dupont A. P. gingivalis in periodontal disease and atherosclerosis - scenes of action for antimicrobial peptides and complement. Front Immunol. 2015;6:45

2. Wimley WC, Hristova K. Antimicrobial peptides: successes, challenges and unanswered questions. J Membr Biol. 2011:239(1-2):27-34.

3. Sang Y, Blecha F. Antimicrobial peptides and bacteriocins: alternatives to traditional antibiotics. Anim Health Res Rev. 2008;9(2):227-35.

4. Shai Y. Mechanism of the binding, insertion and destabilization of phospholipid bilayer membranes by alpha-helical antimicrobial and cell non-selective membrane-lytic peptides. Biochim Biophys Acta. 1999;1462(1-2):55-70.

5. Overhage J, Campisano A, Bains M, Torfs EC, Rehm BH, Hancock RE. Human host defense peptide LL-37 prevents bacterial biofilm formation. Infect Immun. 2008;76(9):4176-82.

6. de la Fuente-Nunez C, Korolik V, Bains M, Nguyen U, Breidenstein EB, Horsman S, Lewenza S, Burrows L, Hancock RE. Inhibition of bacterial biofilm formation and swarming motility by a small synthetic cationic peptide. Antimicrob Agents Chemother. 2012;56(5):2696-704
7. Mackay BJ, Denepitiya L, lacono VJ, Krost SB, Pollock JJ. Growth-inhibitory and bactericidal effects of human parotid salivary histidine-rich polypeptides on Streptococcus mutans. Infect Immun. 1984;44(3):695-701.

8. Rothstein DM, Spacciapoli P, Tran LT, Xu T, Roberts FD, Dalla Serra M, Buxton DK, Oppenheim FG, Friden P. Anticandida activity is retained in P-113, a 12amino-acid fragment of histatin 5. Antimicrob Agents Chemother. 2001;45(5): 1367-73.

9. Sajjan US, Tran LT, Sole N, Rovaldi C, Akiyama A, Friden PM, Forstner JF, Rothstein DM. P-113D, an antimicrobial peptide active against Pseudomonas aeruginosa, retains activity in the presence of sputum from cystic fibrosis patients. Antimicrob Agents Chemother. 2001:45(12):3437-44.

10. Sugiyama K. Anti-lipopolysaccharide activity of histatins, peptides from human saliva. Experientia. 1993:49(12):1095-7.

11. Uitto VJ, Suomalainen K, Sorsa T. Salivary collagenase. Origin, characteristics and relationship to periodontal health. J Periodontal Res. 1990;25(3):135-42.

12. Wang HY, Cheng JW, Yu HY, Lin L, Chih YH, Pan YP. Efficacy of a novel antimicrobial peptide against periodontal pathogens in both planktonic and polymicrobial biofilm states. Acta Biomater. 2015;25:150-61.

13. Yu HY, Tu CH, Yip BS, Chen HL, Cheng HT, Huang KC, Lo HJ, Cheng JW. Easy strategy to increase salt resistance of antimicrobial peptides. Antimicrob Agents Chemother. 2011;55(10):4918-21.

14. Irizarry RA, Hobbs B, Collin F, Beazer-Barclay YD, Antonellis KJ, Scherf U, Speed TP. Exploration, normalization, and summaries of high density oligonucleotide array probe level data. Biostatistics (Oxford, England). 2003;4(2):249-64.

15. Tusher VG, Tibshirani R, Chu G. Significance analysis of microarrays applied to the ionizing radiation response. Proc Natl Acad Sci U S A. 2001:98(9):5116-21.

16. Nelson KE, Fleischmann RD, DeBoy RT, Paulsen IT, Fouts DE, Eisen JA, Daugherty SC, Dodson RJ, Durkin AS, Gwinn M, et al. Complete genome sequence of the oral pathogenic Bacterium porphyromonas gingivalis strain W83. J Bacteriol. 2003;185(18):5591-601.

17. Ribas De Pouplana L, Schimmel P. Aminoacyl-tRNA synthetases: potential markers of genetic code development. Trends Biochem Sci. 2001;26(10):591-6.

18. Hinsa SM, Espinosa-Urgel M, Ramos U, O'Toole GA. Transition from reversible to irreversible attachment during biofilm formation by Pseudomonas fluorescens WCS365 requires an ABC transporter and a large secreted protein. Mol Microbiol. 2003;49(4):905-18.

19. Kolenbrander PE, Andersen RN, Ganeshkumar N. Nucleotide sequence of the Streptococcus gordonii PK488 coaggregation adhesin gene, scaA, and ATP-binding cassette. Infect Immun. 1994:62(10):4469-80.

20. Matthysse AG, Yarnall HA, Young N. Requirement for genes with homology to $A B C$ transport systems for attachment and virulence of Agrobacterium tumefaciens. J Bacteriol. 1996:178(17):5302-8.

21. Diaz PI, Slakeski N, Reynolds EC, Morona R, Rogers AH, Kolenbrander PE. Role of oxyR in the oral anaerobe Porphyromonas gingivalis. J Bacteriol. 2006;188(7):2454-62.

22. McKenzie RM, Aruni W, Johnson NA, Robles A, Dou Y, Henry L, Boskovic DS, Fletcher HM. Metabolome variations in the Porphyromonas gingivalis vimA mutant during hydrogen peroxide-induced oxidative stress. Mol Oral Microbiol. 2015;30(2):111-27.

\section{Submit your next manuscript to BioMed Central and we will help you at every step:}

- We accept pre-submission inquiries

- Our selector tool helps you to find the most relevant journal

- We provide round the clock customer support

- Convenient online submission

- Thorough peer review

- Inclusion in PubMed and all major indexing services

- Maximum visibility for your research

Submit your manuscript at www.biomedcentral.com/submit
Biomed Central 